\title{
CORRECTION
}

\section{Correction to: Imported falciparum malaria in adults: host- and parasite-related factors associated with severity. The French prospective multicenter PALUREA cohort study}

\author{
Fabrice Bruneel ${ }^{1 *}$, Florence Tubach 2,3,4 ${ }^{2}$ Jean-Paul Mira ${ }^{5}$, Sandrine Houze ${ }^{6}$, Sebastien Gibot ${ }^{7}$, \\ Marie-Genevieve Huisse ${ }^{8}$, Bruno Megarbane ${ }^{9}$, Christophe Choquet ${ }^{10}$, Philippe Corne ${ }^{11}$, Eric Peytel ${ }^{12}$, \\ Daniel Villers ${ }^{13}$, Christophe Camus ${ }^{14}$, Olivier Bouchaud ${ }^{15}$, Eric Caumes ${ }^{16}$, Pierre-Marie Girard ${ }^{17}$, Fabrice Simon ${ }^{18}$, \\ Antoine Kalloumeh ${ }^{19}$, Carine Roy ${ }^{3,4}$, Remy Durand ${ }^{20}$, Jacques Le Bras ${ }^{6}$, Sophie Matheron ${ }^{21}$, Michel Wolff22 \\ and The PALUREA Study Group ${ }^{23}$
}

(c) 2017 Springer-Verlag GmbH Germany and ESICM

\section{Correction to: Intensive Care Med (2016) 42:1588- 1596 \\ DOI 10.1007/s00134-016-4356-x}

The members of the Palurea Study Group were provided in such a way that they could not be indexed as collaborators on PubMed. The publisher apologizes for this error and is pleased to list the members of the group here:

Members of the PALUREA Study Group (including authors)

Hopital Bichat Claude Bernard, Paris: M. Wolff, B. Mourvillier, C. Aubron, L. Bouadma and B. Regnier (ICU), S. Matheron, E. d'Ortenzio, R. Matra, E. Bouvet, J. Tourret, J. Dorchies, S. Males, C. Rioux, R. Flicoteaux and P. Yeni (Infectious Diseases Unit), C. Choquet, F. Spingler, E. Kargougou and E. Casalino (Emergency Unit), S. Houze and J. Le Bras (Parasitology and Malaria National Reference Center), M.G. Huisse and V. Ollivier (Hematology), A. Kalloumeh and B. Grandchamp (Biochemistry), F. Tubach, Y. Miri and C. Roy (Département d'Epidémiologie et Recherche Clinique)

\footnotetext{
${ }^{*}$ Correspondence: fbruneel@ch-versailles.fr

'Service de Réanimation Médico-Chirurgicale, Centre Hospitalier de Versailles, 177 rue de Versailles, 78157 Le Chesnay, France Full author information is available at the end of the article

The online version of the original article can be found under doi:10.1007/ s00134-016-4356-x.
} 
Hopital de la Pitie Salpetriere, Paris: J.L. Trouillet, C.E. Luyt, M. Mirabel and J. Chastre (ICU), P. Hochedez, A. Perignon and E. Caumes (Infectious Diseases Unit), M. Thellier, and M. Danis (Parasitology), A. Faussart and J. Nafziger (Biology)

Hopital de Limoges: H. Gastinne and N. Pichon (ICU), D. Ajzenberg and M.L. Darde (Parasitology)

Hopital Antoine Beclere, Clamart: F. Jacobs and F. Brivet (ICU), A. Veyradier and A. Marfaing-Koka (Parasitology and Hematology) 1595

Hopital de Grenoble: J.F. Timsit and L. Hammer (ICU), D. Maubon and H. Pelloux (Parasitology)

Hopital Cochin, Paris: F. Pene, J. Charpentier, S. Marque, C. Rousseau and J.P. Mira (ICU)

Hopital André Mignot, Versailles: F. Bruneel, P. Guezennec, G. Troche, and J.P. Bedos (ICU), O. Eloy and C. Palette (Parasitology and Biology), A. Therby, S. Monnier and A. Greder-Bellan (Infectious Diseases Unit)

Hopital de Rangueil, Toulouse: P. Cougot (ICU)

Hopital Emile Muller, Mulhouse: K. Kuteifan, Y. Mootien and P. Guiot (ICU), J.M. Delarbre and M.H. Kiefer (Microbiology and Parasitology)

Hopital Ambroise Paré, Boulogne: B. Page and A. Vieillard Baron (ICU), J. Dunand (Parasitology)

Hopital de Saint Germain en Laye: Y. Loubieres, JL. Ricome (ICU), J.Y. Peltier and Y. Giudicelli (Parasitology and Biology)

Hopital Begin, Saint Mandé: C. Pelletier and J.M. Rousseau (ICU), P. Imbert, C. Rapp, F. Mechai, R. Barruet and T. Debord (Infectious Diseases Unit), J.E. Pilo and J.D. Cavallo (Biology)

Hopital Purpan, Toulouse: O. Angles and M. Genestal (ICU)

Hopital Hotel Dieu, Nantes: C. Bretonnière, L. Nicolet, L. Jalin and D. Villers (ICU), J.P. Talarmin, O. Grossi and F. Raffi (Infectious Diseases Unit), F. Gay-Andrieu and T. Bompoil (Parasitology)

Hopital Pontchaillou, Rennes: C. Camus, A. Gros and Y. Le Tulzo (ICU), M. Revest, F. Fily, J.M. Chapplain, P. Tattevin and C. Michelet (Infectious Diseases Unit), S. Chevrier and C. Guigen (Parasitology)

Hopital Lariboisiere, Paris: B. Megarbane and F. Baud (ICU), J.M. Launay, C. Gourmel and F. Derouin (Biology and Parasitology)

Hopital Saint Louis, Paris: S. de Miranda, G. Thiery, S. Legriel, E. Azoulay and B. Schlemmer (ICU), N. Colin de Verdieres, M. Lagrange-Xelot, S. Gallien, N. De Castro, J. Pavie, M. Lafaurie and J.M. Molina (Infectious Diseases Unit), C. Sarfati and F. Derouin (Parasitology)

Hopital Gui de Chauliac, Montpellier: P. Corne and P. Jonquet (ICU), J.F. Schved, C. Biron and C. Bret (Hematology)
Hopital Lapeyronie, Montpellier: K. Klouche and P. Beraud (ICU), J.P. Cristol (Biology)

Hopital Pellegrin, Bordeaux: D. Gruson, B. Herpe, M. Delacre and Y. Castaing (ICU), P. Fialon and P. Vincendeau (Parasitology)

Hopital Avicenne, Bobigny: F. Vincent, P. Karoubi and Y. Cohen (ICU), H. Cordel, V. Prendki, J. Cailhol, S. Abgrall, H. Gros, C. Fantinato and O. Bouchaud (Infectious Diseases Unit), F. Cymbalista, Y. Senghor and R. Durand (Parasitology)

Hopital Delafontaine, Saint Denis: N. Memain, M. Thuong, C. Adrie, L. Darques, G. Moret and F. Fraisse (ICU), N. Godineau and C. Chaplain (Parasitology), M. Prevel and R.J. Mary (Emergency Unit)

Hopital Victor Dupouy, Argenteuil: G. Plantefeve, G. Bleichner and H. Mentec (ICU), F. Leturdu (Biology and Parasitology)

Hopital de l'Archet, Nice: H. Hyvernat and G. Bernardin (ICU), P. Delaunay and P. Marty (Parasitology)

Hopital Saint Antoine, Paris: J.L. Baudel, E. Maury and G. Offenstadt (ICU), J.L. Meynard J. Pacanowski, Z. Ouazene, D. Bollens, M.C. Meyohas, L. Fonquerine and P.M. Girard (Infectious Diseases Unit), G. Belkadi, D. Magne and P. Roux (Parasitology)

Hopital Louis Mourier, Colombes: L. Rusel, J.D. Ricard and D. Dreyfuss (ICU), E. Mortier and P. Vinceneux (Infectious Diseases Unit), M. Bloch and G. Galeazzi (Parasitology)

Hopital de la Côte de Nacre, Caen: P. Charbonneau (ICU), C. Duhamel and R. Leclercq (Parasitology)

Hopital d'Aulnay sous-Bois: J.L. Delassus and D. Malbec (Infectious Diseases Unit), D. Lusina and H. Broutier (Parasitology)

Hopital Necker, Paris: C. Charlier, F. Lanternier, H. Coignard and O. Lortholary (Infectious Diseases Unit), M.E. Bougnoux (Parasitology)

Centre Médical de l'Institut Pasteur de Paris: P.H. Consigny and A. Simons de Fanti (Infectious Diseases Unit), A.S. Leguern (Parasitology)

Hôpital de Nancy: S. Gibot (ICU) and F. Massin (Immunology)

\footnotetext{
Author details

1 Service de Réanimation Médico-Chirurgicale, Centre Hospitalier de Versailles, 177 rue de Versailles, 78157 Le Chesnay, France. ${ }^{2}$ Université Paris Diderot, Sorbonne Paris Cité, UMR 1123, 75018 Paris, France. ${ }^{3}$ Département d'Epidémiologie et Recherche Clinique, CIC-EC 1425, AP-HP, Hôpital Bichat, 75018 Paris, France. ${ }^{4}$ INSERM, CIC-EC 1425, U1123, 75018 Paris, France. ${ }^{5}$ Service de Réanimation, Centre Hospitalier Universitaire Cochin Saint-Vincent de Paul, AP-HP, 75014 Paris, France. ${ }^{6}$ Service de Parasitologie, Centre Hospitalier Universitaire Bichat-Claude Bernard, AP-HP, 75018 Paris, France. ${ }^{7}$ Service de Réanimation Médicale, Hôpital Central, Centre Hospitalier Universitaire de Nancy, 54035 Nancy, France. ${ }^{8}$ Unité d'Hémato-Immunologie, Centre Hospitalier Universitaire Bichat-Claude Bernard, AP-HP, 75018 Paris, France. ${ }^{9}$ Service de Réanimation Médicale et Toxicologique, Centre Hospitalier Universitaire Lariboisière, AP-HP, 75010 Paris, France. ${ }^{10}$ Service d'Accueil des Urgences, Centre Hospitalier Universitaire Bichat-Claude Bernard, AP-HP, 75018 Paris, France.
} 
11 Service de Réanimation Médicale, Centre Hospitalier Universitaire Gui de Chauliac, 34295 Montpellier, France. ${ }^{12}$ Service d'Anesthésiologie et Réanimation, Hôpital d'Instruction des Armées Laveran, 13384 Marseille, France. ${ }^{13}$ Service de Réanimation Médicale Polyvalente, Centre Hospitalier Universitaire de Nantes, Hotel Dieu, 44093 Nantes, France. ${ }^{14}$ Service de Réanimation Médicale et Infectieuse, Centre Hospitalier Universitaire de Rennes, Hôpital Pontchaillou, 35033 Rennes, France. ${ }^{15}$ Service de Maladies Infectieuses et Tropicales, Centre Hospitalier Universitaire Avicenne, AP-HP, 93009 Bobigny, France. ${ }^{16}$ Service de Maladies Infectieuses et Tropicales, Centre Hospitalier Universitaire de la Pitié-Salpêtrière, AP-HP, 75013 Paris, France. ${ }^{17}$ Service de Maladies Infectieuses et Tropicales, Centre Hospitalier Universitaire de Saint- Antoine, AP-HP, 75012 Paris, France. ${ }^{18}$ Service de Maladies Infectieuses et Tropicales, Hôpital d'Instruction des Armées Laveran, 13384 Marseille, France. ${ }^{19}$ Unité de Biochimie Clinique, Centre Hospitalier Universitaire Bichat-Claude Bernard, AP-HP, 75018 Paris, France. ${ }^{20}$ Service de Parasitologie, Centre Hospitalier Universitaire Avicenne, AP-HP, 93009 Bobigny, France. ${ }^{21}$ Service de Maladies Infectieuses et Tropicales, Centre Hospitalier Universitaire Bichat-Claude Bernard, AP-HP, 75018 Paris, France. ${ }^{22}$ Service de Réanimation Médicale et Infectieuse, Centre Hospitalier Universitaire Bichat-Claude Bernard, AP-HP, 75018 Paris, France. ${ }^{23}$ Centre Hospitalier Universitaire Bichat-Claude Bernard, AP-HP, 75018 Paris, France.

Published online: 14 November 2017 